\title{
Pyridinethiol-Assisted Dissolution of Elemental Gold in Organic Solutions
}

\author{
Räisänen, Minna
}

2018-12-21

Räisänen , M , Heliövaara , E , Al-Qaisi , F , Muuronen , M , Eronen , A , Liljeqvist , H , Nieger , M , Kemell , M , Moslova , K , Hämäläinen , J , Lagerblom , K \& Repo , T 2018 ,' Pyridinethiol-Assisted Dissolution of Elemental Gold in Organic Solutions ' , Angewandte Chemie (International Edition), vol. 57 , no. 52 , pp. 17104-17109 . https://doi.org/10.1002/anie.201810447

http://hdl.handle.net/10138/324826

https://doi.org/10.1002/anie.201810447

unspecified

acceptedVersion

Downloaded from Helda, University of Helsinki institutional repository.

This is an electronic reprint of the original article.

This reprint may differ from the original in pagination and typographic detail.

Please cite the original version. 
Pyridinethiol-assisted dissolution of elemental gold in organic solutions

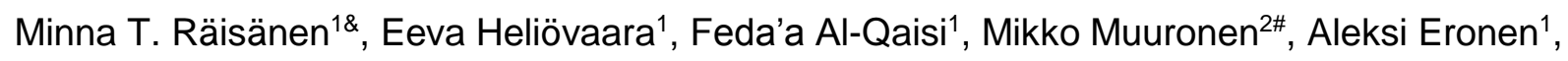
Henri Liljeqvist ${ }^{1}$, Martin Nieger ${ }^{1}$, Marianna Kemell ${ }^{1}$, Karina Moslova ${ }^{1}$, Jani Hämäläinen ${ }^{1}$, Kalle Lagerblom $^{1}$, and Timo Repo ${ }^{1 *}$

\author{
${ }^{1}$ Department of Chemistry, University of Helsinki, P.O. Box 55, FI-00014, Finland. \\ 2Department of Chemistry, University of California, Irvine, 1102 Natural Sciences II, Irvine, \\ California 92697-2025, United States. \\ ${ }^{\&}$ Current address: Academy of Finland, Hakaniemenranta 6, 00530, Finland. \\ \#Current address: BASF SE, Carl-Bosch-Strasse 38, Ludwigshafen, Germany. \\ *e-mail: timo.repo@helsinki.fi
}

Dissolution of elemental $\mathrm{Au}$ in organic solutions is a contemporary approach to lower environmental burden related to gold recycling. Here we describe fundamental studies on a highly efficient method for dissolution of Au which is based on DMF solutions containing pyridine-4-thiol (4-PSH) as a reactive ligand and hydrogen peroxide as an oxidant. Dissolution of Au proceeds mechanistically through several elementary steps: isomerization of 4-PSH to pyridine-4-thione (4-PS); coordination with $\mathrm{Au}^{0}$; then oxidation of $\mathrm{Au}^{0}$ thione species to $\mathrm{Au}^{1}$ simultaneously with the oxidation of free pyridine thione to elementary $S$ and further to sulfuric acid. The final dissolution product is an Au' complex bearing two 4-PS ligands and $\mathrm{SO}_{4}{ }^{2-}$ as a counter ion. The role of the ligand is, therefore, crucial as it assists the oxidation process and stabilizes and solubilizes the formed Au cations. The generality of the designed method was successfully highlighted with other Group 11 metals and its applicability to the circular economy was further demonstrated with printed circuit board samples.

Noble metals, including Au, Ag and Pt, are scarce elements in earth's crust and greatly needed in high-tech applications. ${ }^{1-4}$ They are, therefore, precious and their demand is continuously growing. The possibility to recycle the elements from multi-metal materials such as printed circuit boards (PCBs) is crucial when sustainability and the circular economy are considered. ${ }^{5}$ In general, recovery of $\mathrm{Au}$ in the mining industry is mostly based on the hydrometallurgical cyanidation process, which relies on the use of stoichiometric amounts of cyanide salts and produces large amounts of hazardous waste. ${ }^{6,7}$ Consequently, a new and fairly unexploited concept known as dissolution of noble metals in organic solvents is highly attractive - it offers the possibility to develop benign and selective dissolution methods. ${ }^{8-16}$

Three different methods for dissolution of $\mathrm{Au}^{0}$ in organic solutions have been reported up to date (Fig. 1). One approach is based on pyridine, dimethylformamide or imidazole solutions of thionyl chloride $\left(\mathrm{SOCl}_{2}\right)$ called organic aqua regia (Fig. 1a) which, regardless of the toxicity of $\mathrm{SOCl}_{2}$, 
benefits from high selectivity towards $\mathrm{Pt} / \mathrm{Au} / \mathrm{Pd}$ and $\mathrm{Au} / \mathrm{Pd}$ mixtures. ${ }^{8-10}$ The second method is based on somewhat toxic $\mathrm{I}_{2}$ using dithioxamides or tetraalkylthiuram disulfides as donors to oxidize $\mathrm{Au}^{0}$ to $A u^{\text {III }}$ (Fig. 1b). ${ }^{11-15}$ The third concept is a pyridine-4-thiol (4-PSH) based method developed by us, characterized by slow dissolution of $\mathrm{Au}$ in ethanol solutions $\left(0.06 \times 10^{-3} \mathrm{~mol} /\left(\mathrm{m}^{2 *} \mathrm{~h}\right)\right)$ at room temperature (Fig. 1c).$^{16}$ Interestingly, by fine-tuning the reaction conditions, this method can also be used in selective preparation of self-supporting nanometer-thick gold foils. ${ }^{16}$ As thiols are commonly used as etch resists for $\mathrm{Au}^{0}$ surfaces, the results were intriguing and counterintuitive. Therefore, we took an initiative to carry out further studies and reveal mechanistic details related to the 4-PSHassisted dissolution.

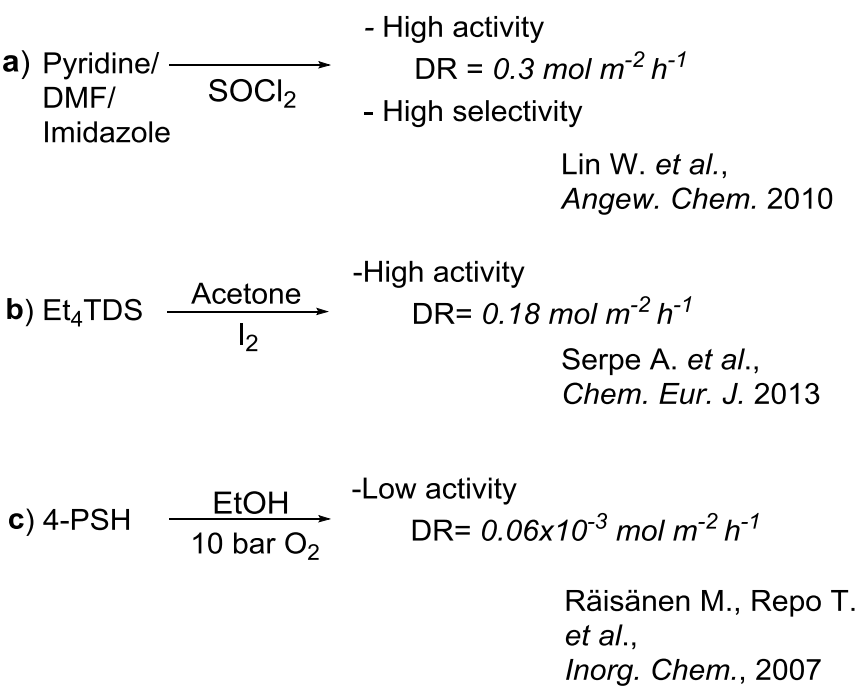

Hydrometallurgical cyanide leaching

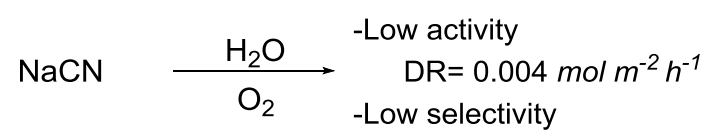

Fig. 1l Organic solvent-based methods for dissolution of elemental gold. a, $\mathrm{SOCl}_{2}$ with $\mathrm{py} / \mathrm{Im} / \mathrm{DMF} \mathbf{b}$, $\mathrm{Et}_{4} \mathrm{TDS} / \mathrm{I}_{2} \mathbf{c}, 4-\mathrm{PSH} / \mathrm{EtOH} / \mathrm{O}_{2}$ and comparison with common hydrometallurgical cyanide leaching. DR stands for dissolution rate.

Here we report a new dissolution method for elemental Au that rapidly and quantitatively dissolves Au upon heating in DMF solution of 4-PSH and $\mathrm{H}_{2} \mathrm{O}_{2}$. The achieved rate, $0.10 \mathrm{~mol} /\left(\mathrm{m}^{2 *} \mathrm{~h}\right)$, is 1,600 fold higher than that measured for the published 4-PSH protocol ${ }^{16}$, and comparable in efficiency to the other two non-conventional methods. ${ }^{8,15}$ Experimental and computational studies revealed mechanistic insights for the dissolution of $\mathrm{Au}$, particularly the role for 4-PSH and the selectivity of the system towards metals with the stable oxidation state +1 . In terms of the circular economy, the study opens opportunities to develop technologically and economically viable dissolution of elemental gold. 


\section{Results and discussion}

New dissolution method for Au powder. The study was initiated by conducting Au dissolution experiments using our previously published protocol ${ }^{16}$ as follows: a small amount of fine Au powder was added to $16 \mathrm{mM} \mathrm{EtOH}$ solution of 4-PSH and the mixture was stirred for $23 \mathrm{~h}$ under 10 bar of $\mathrm{O}_{2}$ at $22^{\circ} \mathrm{C}$. This resulted in only $14 \%$ of dissolved Au (Table 1, entry 1 ). To increase Au dissolution, a series of optimization reactions was carried out varying the solvent, oxidant, ligand concentration and temperature (see Supplementary Section 2). As a result, we found that Au could be quantitatively dissolved upon heating at $60^{\circ} \mathrm{C}$ in DMF solutions of 4-PSH using $\mathrm{H}_{2} \mathrm{O}_{2}$ as an oxidant (Table 1, entry 2). A noteworthy interdependence between solvent and oxidant was observed, since a similar increase in the dissolution efficiency could not be achieved by changing either the solvent or oxidant alone. Next, a series of experiments was performed with gold powder in DMF solutions with 4-PSH concentrations of 20,102, 203 and $406 \mathrm{mM}$, resulting in 1\%,50\%, 67\% and 84\% of dissolved $\mathrm{Au}$ in only $8 \mathrm{~min}$ (Table 1, entries 3-6) showing that the dissolution efficiency correlates directly with the 4-PSH concentration. Importantly, complete dissolution of Au sample was achieved either with extended reaction time (23h) and low 4-PSH concentration or within 25 min using 203 $\mathrm{mM}$ 4-PSH concentration (Table 1, entries 2 and 7). The latter result is equivalent to an Au dissolution rate of $0.06 \mathrm{~mol} /\left(\mathrm{m}^{2 *} \mathrm{~h}\right)$, which is 1,000 -fold higher than that obtained under the initial conditions which utilized $\mathrm{O}_{2}$ (10 bar) as oxidant in $\mathrm{EtOH}$ solution at $\mathrm{RT}^{16}$.

To obtain an understanding of the reaction mechanism, the partial composition of the reaction solution was studied during the dissolution. According to ${ }^{1} \mathrm{H}$ NMR studies, pyridine-4-thiol (4-PSH) is prone to isomerize to pyridine-4-thione (4-PS) which is the major component in the studied $d_{7^{-}}$ DMF solutions (Fig. 2, Step I, Supplementary Figs. S4-S5). This is in accordance with our previous studies on 4-PSH in alcohol solutions ${ }^{16}$ where a similar equilibrium was observed. We assume that the isomerization of 4-PSH is highly beneficial for 4-PS coordination to $\mathrm{Au}^{0}$ surfaces and, in this way, for initiating the dissolution. Upon heating the solution to $60^{\circ} \mathrm{C}$ and introducing $\mathrm{H}_{2} \mathrm{O}_{2}$ as an oxidant, signals belonging to 4-PS disappear and signals belonging to 4,4'-dipyridyl sulfide 2, 4,4'-dipyridyl disulfide 3 and 4,4'-dipyridyl trisulfide 4 appear in the ${ }^{1} \mathrm{H}$ NMR spectrum (Fig. 2, step II, Supplementary Figs. S6-S7). This was also confirmed by HRMS studies wherein transformation of 4-PSH to 2, 3 and 4 was clearly established (Supplementary Figs. S28-S33). At the same time, HRMS analysis of the reaction mixture revealed that $\left[\mathrm{Au}^{\prime}(4-\mathrm{PS})_{2}\right]^{+} \mathbf{1}$ is the main gold cation in the solution. In addition to 1, Au' complexes 5 and 6 bearing 2 and 4-PS ligands were identified with HRMS (Fig. 2, step III, Supplementary Figs. S34-S37).

We were able to isolate 1 in crystalline form with the $\mathrm{SO}_{4}{ }^{2-}$ as the counter anion and use XRD for structural analysis (see Supplementary Section 3.3 for details). Accordingly, the $\mathrm{Au}^{\prime}$ centers coordinate two neutral 4-PS ligands and $\mathrm{SO}_{4}{ }^{2-}$ balances the overall charge of two adjacent $\mathrm{Au}^{\prime}$ complexes (Fig. 2). In the solid state, the dimeric structure is further stabilized by a weak aurophilic 
interaction (3.1314 $\AA$ ). Together with formation of 1 in the dissolution reaction, a significant amount of yellow crystals also precipitate from the DMF solution after prolonged storage. Upon XRD analysis, the crystals were identified as elemental sulfur $\left(\mathrm{S}_{8}\right)$. In fact, it is known that oxidized sulfur species are formed during the self-assembly of $4-\mathrm{PSH}^{16}$ or alkane thiols ${ }^{17-18}$ on gold surfaces. As strongly oxidizing conditions are needed for efficient dissolution of elementary gold, $S_{8}$ might have an important role; it can react with $\mathrm{H}_{2} \mathrm{O}_{2}$ and form $\mathrm{SO}_{2}$ and successively $\mathrm{SO}_{3}$, which then generates sulfuric acid in contact with $\mathrm{H}_{2} \mathrm{O}$ (Fig. 2, step IV). The combination of sulfuric acid and $\mathrm{H}_{2} \mathrm{O}_{2}$ is thought to form a strong enough oxidant, an in situ made Piranha-like solution, to assist the oxidation of $\mathrm{Au}^{0}$ to $\mathrm{Au}^{\prime}$, where the generated $\mathrm{SO}_{4}{ }^{2-}$ anions serve as a counter ion (Fig. 2).

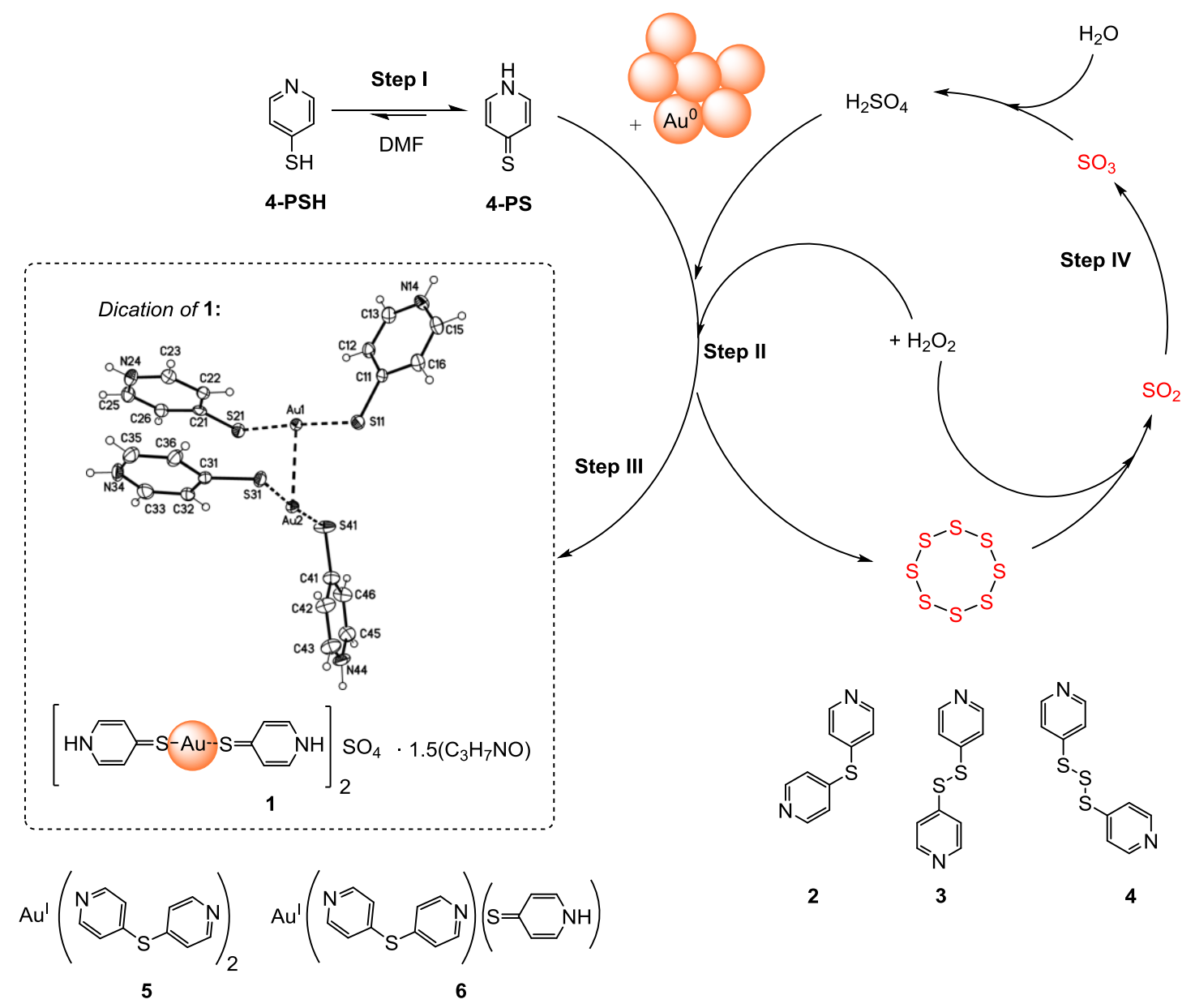

Fig. 2I Elementary reaction steps during the dissolution of elemental Au. Step I: isomerization of pyridine4-thiol to pyridine-4-thione and coordination with the $\mathrm{Au}^{0}$ surface, Step II/III: oxidation of $\mathrm{Au}^{0}$ to $\mathrm{Au}^{\mathrm{l}}$ and simultaneous oxidation of free pyridine-4-thione. Aul species detected in HRMS during the dissolution are $\mathrm{Au}^{\prime}(\text { pyridine-4-thione })_{2}{ }^{+}$1, (Au'(dipyridyl sulfide) $2^{+} \mathbf{5}$ and $\left(\mathrm{Au}^{\prime}(\text { dipyridyl sulfide)(pyridine-4-thione })\right)^{+} \mathbf{6}$. A solidstate structure of 1 [bis(pyridine-4-thione)Au] $\mathrm{SO}_{4}$ illustrates a linear coordination sphere of $\mathrm{Au}^{\prime}$ and a weak aurophilic interaction. The sulfate anion and DMF molecules are omitted from the X-ray structure. Several 4PSH derived products were detected simultaneously with oxidized Au' species including 4,4'-dipyridyl sulfide 2, 4,4'-dipyridyl disulfide 3, 4,4'-dipyridyl trisulfide 4 and elementary sulfur ( $\left.\mathrm{S}_{8}\right)$. Step IV: further oxidation of $\mathrm{S}_{8}$ to sulfuric acid. 
Evidently, due to the marked excess of 4-PSH needed for efficient dissolution and the observed sulfate counter anion of the $\mathrm{Au}$ ' complex - the relation between in situ formed $\mathrm{S}_{8}$ and the dissolution rate of $A u$ appeared to be important. To confirm this hypothesis, we attempted to use external $S_{8}$ as an accelerator by introducing one equivalent of $S_{8}$ into the reaction. With this approach, the dissolution of Au powder with high 4-PSH concentrations (406 and $203 \mathrm{mM}$ ) and external $\mathrm{S}_{8}$ had a minor effect on the dissolution efficiency (Table 1, entries 8 and 9). In contrast, when the concentration of 4-PSH was markedly reduced $(20 \mathrm{mM})$, the added $\mathrm{S}_{8}$ greatly enhanced the dissolution of $\mathrm{Au}$ (Table 1, entries 3 and 4 vs. 10 and 11). These results imply that the dissolution reaction depends strongly on the presence of $\mathrm{S}_{8}$. With low 4-PSH concentrations there is less free ligand and in situ formation of $\mathrm{S}_{8}$ is the rate limiting factor. Notably, no rise in the dissolution efficiency was observed with the above stoichiometric amounts of $S_{8}$ (see Supplementary Section 3.4 for details).

Table 1 I Optimization of dissolution conditions for Au powder by varying the 4-PSH concentration, oxidant, solvent, time and additive $S_{8}$.

\begin{tabular}{lcccccc} 
Entry & Solvent & Oxidant & c [4-PS $](\mathrm{mM})$ & Time & Temperature $\left({ }^{\circ} \mathrm{C}\right)$ & Dissolved Au (\%) \\
\hline 1 & EtOH & $\mathrm{O}_{2}$ & 16 & $23 \mathrm{~h}$ & 22 & $14^{\mathrm{a}}$ \\
2 & $\mathrm{DMF}$ & $\mathrm{H}_{2} \mathrm{O}_{2}$ & 16 & $23 \mathrm{~h}$ & 60 & $99^{\mathrm{a}}$ \\
3 & $\mathrm{DMF}$ & $\mathrm{H}_{2} \mathrm{O}_{2}$ & 20 & $8 \mathrm{~min}$ & 60 & $1^{\mathrm{b}}$ \\
4 & $\mathrm{DMF}$ & $\mathrm{H}_{2} \mathrm{O}_{2}$ & 102 & $8 \mathrm{~min}$ & 60 & $50^{\mathrm{b}}$ \\
5 & $\mathrm{DMF}$ & $\mathrm{H}_{2} \mathrm{O}_{2}$ & 203 & $8 \mathrm{~min}$ & 60 & $67^{\mathrm{b}}$ \\
6 & $\mathrm{DMF}$ & $\mathrm{H}_{2} \mathrm{O}_{2}$ & 406 & $8 \mathrm{~min}$ & 60 & $84^{\mathrm{b}}$ \\
7 & $\mathrm{DMF}$ & $\mathrm{H}_{2} \mathrm{O}_{2}$ & 203 & $25 \mathrm{~min}$ & 60 & $99^{\mathrm{c}}$
\end{tabular}

With added $\mathrm{S}_{8}$

$\begin{array}{lllllll}8 & \text { DMF } & \mathrm{H}_{2} \mathrm{O}_{2} & 406 & 8 \min & 60 & 86^{\mathrm{d}} \\ 9 & \mathrm{DMF} & \mathrm{H}_{2} \mathrm{O}_{2} & 203 & 8 \mathrm{~min} & 60 & 73^{\mathrm{d}} \\ 10 & \mathrm{DMF} & \mathrm{H}_{2} \mathrm{O}_{2} & 102 & 8 \mathrm{~min} & 60 & 61^{\mathrm{d}} \\ 11 & \mathrm{DMF} & \mathrm{H}_{2} \mathrm{O}_{2} & 20 & 8 \mathrm{~min} & 60 & 33^{\mathrm{d}} \\ 12 & \mathrm{DMF} & \mathrm{H}_{2} \mathrm{O}_{2} & 20 & 8 \mathrm{~min} & 60 & 30^{\mathrm{e}}\end{array}$

* Au concentration analyzed by flame atom absorption spectroscopy (FAAS).

aConditions: $1 \mathrm{mg}$ of Au powder (5 $\mu \mathrm{mol}$, particle size 1.5-3.0 $\mu \mathrm{m}$ ), $27 \mathrm{mg}$ of 4-PSH (243 $\mu \mathrm{mol}, \mathrm{c}[4-\mathrm{PSH}]=16$ $\mathrm{mM}), \mathrm{H}_{2} \mathrm{O}_{2}$ (46,4 $\mu$ l, when applicable), $\mathrm{O}_{2}$ (10 bar, when applicable) DMF (15 ml), $23 \mathrm{~h}$.

bConditions: $2 \mathrm{mg}$ of Au powder (10 $\mu \mathrm{mol}$, particle size 1.5-3.0 $\mu \mathrm{m}), \mathrm{H}_{2} \mathrm{O}_{2}(92.8 \mu \mathrm{l})$, DMF (5 ml), 8 min. c[4$\mathrm{PSH}]=102,203$ or $406 \mathrm{mM}$.

'Conditions: Au thin film (300 nm), $294 \mathrm{mg}$ of 4-PSH (c[4-PS] = $203 \mathrm{mM}), 1.7 \mathrm{mg}$ of $\mathrm{S}_{8}, \mathrm{H}_{2} \mathrm{O}_{2}(61 \mu \mathrm{l})$, DMF (13 $\mathrm{ml})$. Mass of Au ca. $1.3 \mathrm{mg}$.

dConditions: as in $\mathrm{b}$, but $\mathrm{Au}: \mathrm{S}_{8}=1: 1$.

eConditions: as in $\mathrm{b}$, but $\mathrm{Au}: \mathrm{S}_{8}=1: 4$. 
Insights into the dissolution mechanism. The dissolution mechanism was further investigated in a combined experimental/theoretical study. To determine the ligand dependence of the gold dissolution, experiments with 4-PSH, 2-pyridinethiol (2-PSH), 4,4'-dipyridyl disulfide (3) and 2mercaptobenzimidazole (2-MBIH) were conducted under the same reaction conditions. At $60{ }^{\circ} \mathrm{C}$ and with 200-fold excess of the ligand, 4-PSH dissolves $81 \%$, both 2-PSH and 3 dissolve $11 \%$ and 2MBIH dissolves $8 \%$ of Au. According to ${ }^{1} \mathrm{H}$ NMR and HRMS analysis of the reaction mixtures, with 2-PSH the thione/thiol equilibrium resides on the thione side. In the presence of the oxidant, 2-PSH is readily oxidized to 2,2'-dipyridyl disulfide which is the major component in the solution (Supplementary Figs. S15-S20). Interestingly, in the presence of $\mathrm{H}_{2} \mathrm{O}_{2}$, neither 3 nor 2-MBIH produce sulfides in amounts that are detectable by ${ }^{1} \mathrm{H}$ NMR (see Supplementary Section 3.1.2 for details). The slow formation of $S_{8}$ from thiol ligands could partially explain the very low dissolution capability of 2-PSH, 3 and 2-MBIH; but the use of $\mathrm{S}_{8}$ as an additive does not improve the Au dissolution rate (Supplementary Section 3.4). For example, 2-MBIH gives a similar $8 \%$ Au dissolution with and without $S_{8}$ in otherwise similar reaction conditions. This result implies that the dissolution of Au with 2-MBIH does not rely on rapid oxidation of thiol ligand to $S_{8}$. Instead, it seems likely that the dissolution rate is dependent on the coordination ability of the thione isomer of the ligands to the gold surface and on the oxidation potential of the formed reactive intermediate.

The ligand influence on the one electron oxidation of $\mathrm{Au}^{0}$ was computationally rationalized following the suggested mechanism that the dissolution is initiated by ligand coordination (Table 2). In principle, gold could be present in solution as mono- or bisligand complexes, although only the latter is experimentally observed. According to the DFT calculations, the coordination of the second ligand to the monoligand Au complex lowers the oxidation potential of $\mathrm{Au}^{0}, 1.2 \mathrm{~V}$ with 4-PSH and $1.6 \mathrm{~V}$ with 2-MBIH (Table 2, entries 1 and 3, Supplementary Section 8.1). Thus it seems that the formation of bisligand $\mathrm{Au}^{0}$ centers is crucial for the oxidation reaction. Such $\mathrm{Au}^{0}$ species are most likely formed at the corners and edges of the gold surface, thus rationalizing a similar etching order observed in experiments with $\mathrm{Au}$ thin films (Supplementary Section 4). According to the calculations, the significant lowering of the oxidation potential of bisligand $\mathrm{Au}^{0}$ species is caused by delocalization of the $6 s^{1}$ electron on the ligands, thus allowing the one-electron oxidation of the ligand instead of the metal. This theoretical insight further supports the experimental results which show that $\mathrm{Pd}$ and $\mathrm{Pt}$ were not dissolved with pyridinethiol ligands as their dissolution would require two-electron oxidation (Supplementary Section 5.2). 
Table 2I Calculated oxidation potentials for mono- and bisligand Au complexes and the formation energies of bisligand complexes from monoligand ones. ${ }^{c}$

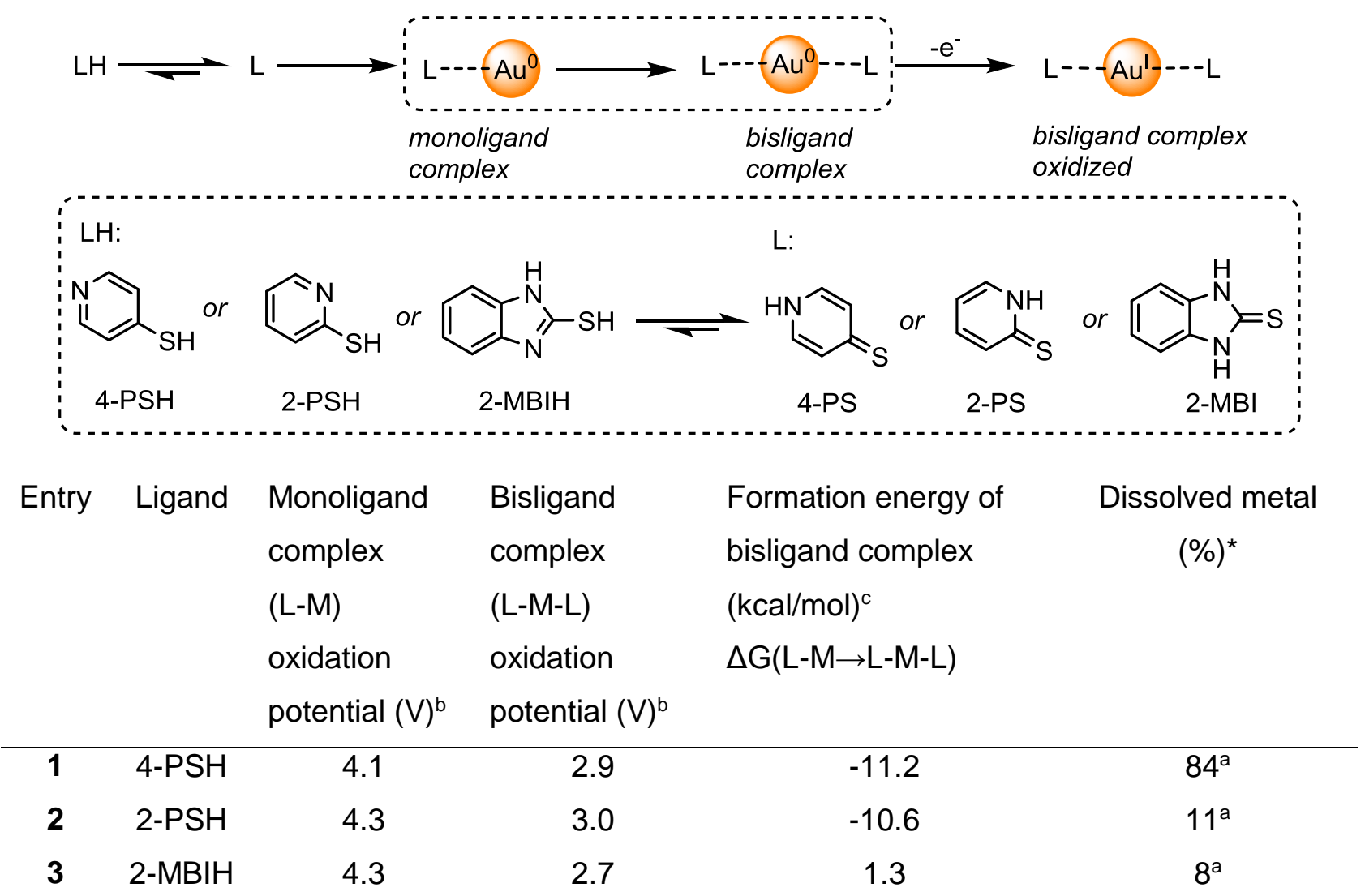

* Experimental reaction conditions: DMF $(5 \mathrm{ml}), 60^{\circ} \mathrm{C}, 8 \mathrm{~min}$, molar ratio: $\mathrm{M} / \mathrm{LH} / \mathrm{H}_{2} \mathrm{O}_{2}$ 1:200:100. Metal concentrations analyzed by FAAS.

a $\mathrm{Au}^{0}$ (2 mg, $0.010 \mathrm{mmol}$ ), 4-PSH (226 mg, $2.033 \mathrm{mmol}$ ) or 2-PSH (226 mg, $2.033 \mathrm{mmol}$ ) or 2-MBIH (305 mg, $2.033 \mathrm{mmol}), \mathrm{H}_{2} \mathrm{O}_{2}(92.6 \mu \mathrm{l})$, DMF $(5 \mathrm{ml})$.

${ }^{b}$ Computed absolute oxidation potentials (in V) at TPSS-D3/def2-TZVP/COSMO levels.

${ }^{c}$ Formation free energies of the bisligand metal complexes from the monoligand complexes at TPSS-D3/def2TZVP/COSMO (left) levels. Energies are in $\mathrm{kcal} / \mathrm{mol}$.

The calculations also give an interesting insight into the markedly lower reactivity of 2-PSH and 2$\mathrm{MBIH}$ in comparison to 4-PSH in the dissolution of $\mathrm{Au}$. The oxidation potentials of reactive intermediates increase in the order $(2-\mathrm{MBI})_{2} \mathrm{Au}^{0}<(4-\mathrm{PS})_{2} \mathrm{Au}^{0}<(2-\mathrm{PS})_{2} \mathrm{Au}^{0}$ and the significantly lower reactivity of 2-PSH in the dissolution of $\mathrm{Au}$ can be rationalized by considering the $0.1 \mathrm{~V}$ higher oxidation potential of (2-PS) ${ }_{2} \mathrm{Au}^{0}$ compared with the (4-PS) ${ }_{2} \mathrm{Au}^{0}$ complex (Table 2, entries 1-3). On the other hand, the $(2-\mathrm{MBI})_{2} \mathrm{Au}^{0}$ complex exhibits a $0.2 \mathrm{~V}$ lower oxidation potential than (4-PS $)_{2} \mathrm{Au}^{0}$ but its endothermic formation energies hamper the overall dissolution reaction (Table 2, entry 3 ). Unambiguously, the rate of dissolution is dependent on the formation energy of the bisligand intermediate and its oxidation potential. 
Dissolution of other coinage metals. 4-PSH assisted dissolution can also be successfully applied to other coinage metals, namely silver and copper, i.e. metals with the stable oxidation state of +1 . Using a high 4-PSH concentration $(\mathrm{c}[4 \mathrm{PSH}]=742 \mathrm{mM})$ at $60^{\circ} \mathrm{C}$, the reaction was very fast and after $8 \mathrm{~min} 61 \%$ of $\mathrm{Ag}$ and $72 \%$ of $\mathrm{Cu}$ were dissolved (Table 3, entries 1 and 3). When 4-PSH loading was reduced, the reaction slowed down and with 10-fold ligand excess only minor amounts of the metals dissolved (4\% Cu, 17\% Ag). Yet a significant accelerating effect was observed for $\mathrm{Cu}$ when the experiment were repeated with $\mathrm{S}_{8}$ additive in otherwise similar dissolution conditions $(20 \% \mathrm{Cu}$, see Supplementary Section 5.4). To identify the structures of the resulting $\mathrm{Ag}^{\prime}$ and $\mathrm{Cu}^{\prime}$ complexes, the products were isolated and analyzed with FTIR, TGA and elemental analysis (EA). Based on the results, the structures of $\mathrm{Cu}^{\prime}$ complex 9 and $\mathrm{Ag}^{\prime}$ complex 10 resemble that of 1 . The counter anion in $\mathbf{9}$ and $\mathbf{1 0}$ can be clearly identified from FTIR spectra as the observed broad peaks around wave numbers $1100 \mathrm{~cm}^{-1}$ (strong) and $600 \mathrm{~cm}^{-1}$ are characteristic of the $v_{3}$ and $v_{4}$ vibrational modes of $\mathrm{SO}_{4}{ }^{2-}$, respectively ${ }^{19-22}$ (Supplementary Section 5.5). According to EA (CHNS) and TGA, the Cu' center binds two 4-PS ligands and a sulfate anion stabilizes the overall charge of the dimeric structure, while each $\mathrm{Ag}^{\prime}$ center binds only one 4-PS ligand and $\mathrm{SO}_{4}{ }^{2-}$ serves as the counter anion.

Next, dissolution experiments with 2-PSH and 2-MBIH were performed for $\mathrm{Cu}^{0}$ and $\mathrm{Ag}^{0}$ similarly as before for $\mathrm{Au}^{0}$; $\mathrm{Cu}$ and $\mathrm{Ag}$ powders were hardly affected by 2-PSH solutions while reactions with high 2-MBIH concentration dissolved $\mathrm{Ag} 15 \%$ and $\mathrm{Cu} 78 \%$ in 8 min reaction time. When the ratio of 2-MBIH to $\mathrm{Cu}^{0}$ was reduced from 200:1 to $10: 1$ and $\mathrm{S}_{8}$ was used as an additive, $\mathrm{Cu}^{0}$ was dissolved $48 \%$ in 8 min reaction time and quantitatively in elongated 30 min reaction time (Supplementary Section 5.4).

The reactivity differences between 2-MBIH with $\mathrm{Cu}^{0}$ and $\mathrm{Au}^{0}$ demonstrate a preferred dissolution selectivity among the coinage metals. This was further investigated with theoretical studies on the dissolution of $\mathrm{Cu}^{0}$ and $\mathrm{Ag}^{0}$ with 4-PSH and 2-MBIH ligands. As observed with the Au complexes, the coordination of the second 4-PS ligand markedly lowers the oxidation potential of $\mathrm{Cu}^{0}$ and $\mathrm{Ag}^{0}$ and delocalization of the $4 s^{1} / 5 s^{1}$ electron on the ligands favors the one-electron oxidation of the metal (Table 3, entries 1 and 3). Due to the $0.1 \mathrm{~V}$ lower oxidation potential of $(4-\mathrm{PS})_{2} \mathrm{Cu}^{0}$ and $(4-\mathrm{PS})_{2} \mathrm{Ag}^{0}$ species compared to (4-PS) ${ }_{2} \mathrm{Au}^{0}$, they are predicted to be oxidized slightly faster. Although the theoretical results support the fast dissolution of metallic $\mathrm{Ag}$ and $\mathrm{Cu}$, it seems that the true efficiency of the dissolution method is hampered e.g. by formation of an oxide layer on the metal surface. This leads unexpectedly to a higher experimental dissolution efficiency with Au. From the extended ligand series, $(2-\mathrm{MBI})_{2} \mathrm{Cu}^{0}$ and $(2-\mathrm{MBI})_{2} \mathrm{Ag}^{0}$ species exhibit lower oxidation potentials than their 4-PS analogues, but the dissolution reactivity towards $\mathrm{Ag}^{0}$ (as with $\mathrm{Au}^{0}$ above) is reduced by endothermic complex formation energies (Table 3 , entry 2$)$. $(2-\mathrm{MBI})_{2} \mathrm{Cu}^{0}$ has an exothermic formation energy and shows a high dissolution rate. This rationalized the experimentally observed minor dissolution of $\mathrm{Ag}^{0}$ with $2-\mathrm{MBIH}$ assisted reactions while $\mathrm{Cu}^{0}$ is efficiently dissolved. 
Table 3l Calculated oxidation potentials for mono- and bisligand $\mathrm{Ag}$ and $\mathrm{Cu}$ complexes and the formation energies of bisligand complexes from monoligand ones. ${ }^{d}$

\begin{tabular}{|c|c|c|c|c|c|c|}
\hline Entry & Ligand & Metal & $\begin{array}{l}\text { Monoligand } \\
\text { complex } \\
(\mathrm{L}-\mathrm{M}) \text { oxidation } \\
\text { potential }(\mathrm{V})^{\mathrm{C}}\end{array}$ & $\begin{array}{l}\text { Bisligand } \\
\text { complex } \\
(\mathrm{L}-\mathrm{M}-\mathrm{L}) \\
\text { oxidation } \\
\text { potential }(\mathrm{V})^{\mathrm{C}}\end{array}$ & $\begin{array}{l}\text { Formation energy of } \\
\text { bisligand complex } \\
(\mathrm{kcal} / \mathrm{mol})^{\mathrm{d}} \\
\Delta \mathrm{G}(\mathrm{L}-\mathrm{M} \rightarrow \mathrm{L}-\mathrm{M}-\mathrm{L})\end{array}$ & $\begin{array}{c}\text { Dissolved } \\
\text { metal } \\
(\%)^{*}\end{array}$ \\
\hline 1 & 4-PSH & $\mathrm{Ag}$ & 3.5 & 2.8 & -8.5 & $61^{a}$ \\
\hline 2 & 2-MBIH & $\mathrm{Ag}$ & 3.6 & 2.7 & 3.0 & $15^{\mathrm{a}}$ \\
\hline 3 & 4-PSH & $\mathrm{Cu}$ & 3.4 & 2.8 & -19.6 & $72^{b}$ \\
\hline 4 & 2-MBIH & $\mathrm{Cu}$ & 3.6 & 2.5 & -5.6 & $78^{b}$ \\
\hline
\end{tabular}

* Reaction conditions: DMF (5 ml), 60 ${ }^{\circ} \mathrm{C}, 8 \mathrm{~min}$, molar ratio: $\mathrm{M} / \mathrm{LH} / \mathrm{H}_{2} \mathrm{O}_{2}$ 1:200:100. Metal concentrations analyzed by FAAS.

a $\mathrm{Ag}^{0}$ (2 mg, $0.019 \mathrm{mmol}$ ), 4-PSH (412 mg, $3.709 \mathrm{mmol}$ ) or 2-PSH (412 mg, $3.709 \mathrm{mmol}$ ) or 2-MBIH (557 mg, $3.709 \mathrm{mmol}), \mathrm{H}_{2} \mathrm{O}_{2}(172 \mu \mathrm{l})$, DMF $(5 \mathrm{ml})$.

${ }^{b} \mathrm{Cu}^{0}(2 \mathrm{mg}, 0.032 \mathrm{mmol}), 4-\mathrm{PSH}(700 \mathrm{mg}, 6.297 \mathrm{mmol})$ or 2-PSH (700 mg, $\left.6.297 \mathrm{mmol}\right)$ or 2-MBIH (945 mg, $6.297 \mathrm{mmol}), \mathrm{H}_{2} \mathrm{O}_{2}(292 \mu \mathrm{l})$, DMF $(5 \mathrm{ml})$.

c Computed absolute oxidation potentials (in V) at TPSS-D3/def2-TZVP/COSMO levels.

${ }^{d}$ Formation free energies of the bisligand metal complexes from the monoligand complexes at TPSS-D3/def2TZVP/COSMO (left) levels. Energies are in $\mathrm{kcal} / \mathrm{mol}$.

Dissolution of $\mathrm{Au}$ in thin films and printed circuit boards (PCB). Recycling of noble metals from secondary sources including electronic waste is becoming increasingly important when modern societies are heading towards a circular economy. In this respect, dissolution of Au films is essential and methods other than cyanidation or $\mathrm{Hg} / \mathrm{Au}$ amalgam extractions are also needed to fulfill the criteria of green chemistry and sustainability. ${ }^{23}$ Due to their lower surface area, Au films are more challenging substrates than powders. We studied the dissolution of $300 \mathrm{~nm}$ thick Au films $(1 \mathrm{~cm} \times$ $2.2 \mathrm{~cm}$ on mica substrate) and noted that etching began at the edges and then steadily progressed towards the center of the film (Fig. 3a). The studied Au film was fully dissolved in a DMF solution of 4- $\mathrm{PSH} / \mathrm{H}_{2} \mathrm{O}_{2}(\mathrm{c}[\mathrm{PSH}]=203 \mathrm{mM})$ in $18 \mathrm{~min}$, which corresponds to an Au dissolution rate of 0.06 $\mathrm{mol} /\left(\mathrm{m}^{2 *} \mathrm{~h}\right)$. Remarkably, with added $\mathrm{S}_{8}$, an even more efficient dissolution rate was achieved $(0.10$ $\mathrm{mol} /\left(\mathrm{m}^{2 *} \mathrm{~h}\right)$ corresponding to a 1,600-fold enhancement when compared to our previous 4-PSH/EtOH method $\left(0.06 \times 10^{-3} \mathrm{~mol} /\left(\mathrm{m}^{2 *} \mathrm{~h}\right)\right)^{16}$.

Because of their multi-metallic composition, we considered PCBs as one of the most challenging substrates and identified the so-called 'Au finger' part suitable for dissolution studies. Accordingly, this type of sample contains $23.4 \mathrm{~m}-\%$ of $\mathrm{Cu}$ and only $0.25 \mathrm{~m}-\%$ of $\mathrm{Au}$ (and $0.008 \mathrm{~m}-\%$ of $\mathrm{Ag}$ ) (see Supplementary Section 6 for details). In this regard, a direct 4-PSH leaching of Au is not rational due 
to the high $\mathrm{Cu}$ content compared to that of $\mathrm{Au}$. Therefore, we treated the sample first with $\mathrm{NH}_{3} /\left(\mathrm{NH}_{4}\right)_{2} \mathrm{SO}_{4}$ and $\mathrm{H}_{2} \mathrm{O}_{2}$ to extract $\mathrm{Cu}$ and $\mathrm{Ag}^{24}$ (Fig. 3b, step I). The residue was then filtered and immersed in a DMF solution of $4-\mathrm{PSH} / \mathrm{H}_{2} \mathrm{O}_{2}$ resulting in a significant $84 \%$ of dissolved $\mathrm{Au}$ (Fig. 3b, step II). As the 4-PSH-assisted dissolution method is selective for Group 11 metals, the recovery of these metals from Pt and Pd mixtures is also possible.

a

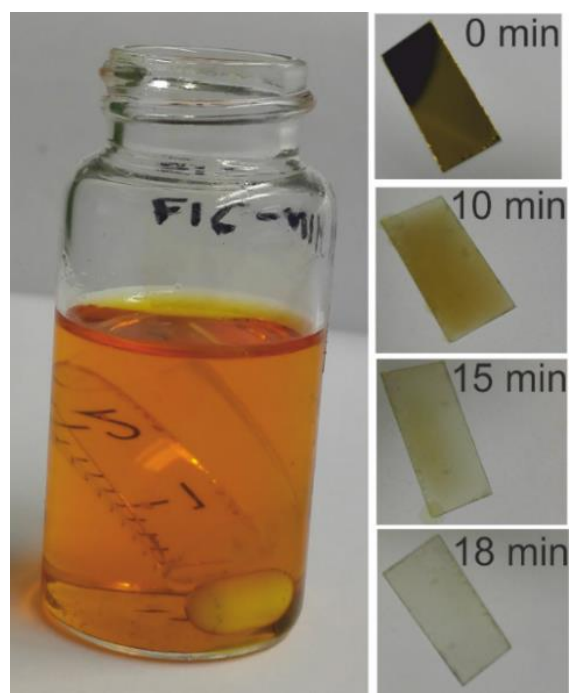

b

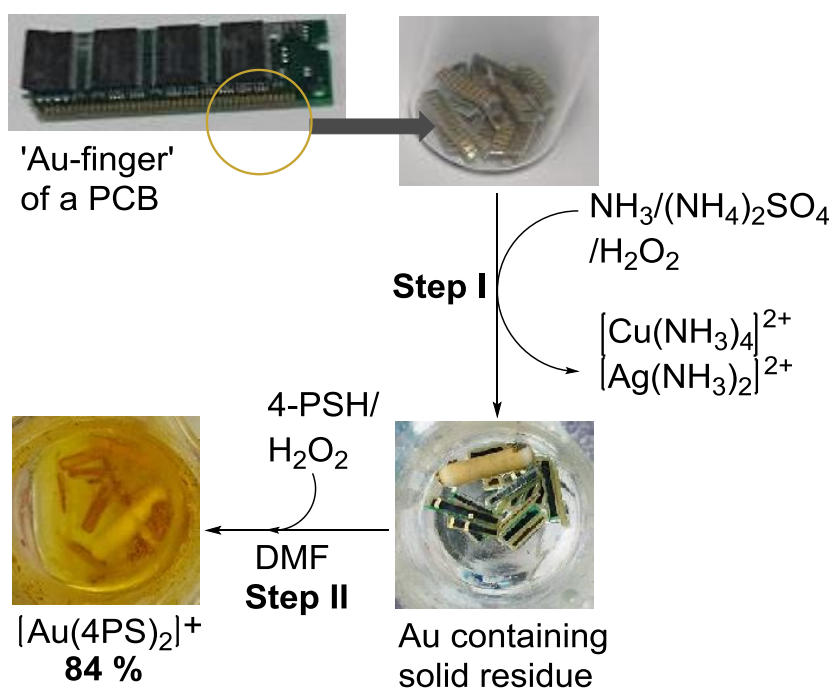

Fig. 3I Dissolution of Au from thin film substrates and PCB samples. a, Progress of $A u$ thin film etching. On the left; a reaction setup used to protect the Au film from mechanical peeling and, on the right; the progress of the etching process from edges towards center of the film. $\mathbf{b}$, Dissolution of Au in PCB samples. Step I: extraction of $\mathrm{Cu}$ and $\mathrm{Ag}$ with $\mathrm{NH}_{3} /\left(\mathrm{NH}_{4}\right)_{2} \mathrm{SO}_{4} / \mathrm{H}_{2} \mathrm{O}_{2}$ and Step II: dissolution of Au with 4-PSH$/ \mathrm{H}_{2} \mathrm{O}_{2}$ method.

\section{Conclusions}

4-PSH as a reactive ligand and hydrogen peroxide as an oxidant forms together a highly efficient combination for dissolution of metallic gold. The reaction is driven by the ability of a thione isomer of 4-PSH to coordinate to a gold surface and to lower oxidation potentials of the gold atoms on the surface. Simultaneously, a part of $4-\mathrm{PSH}$ is oxidized to $\mathrm{S}_{8}$ and to the sulfate anions needed in the reaction to solubilize the generated $\mathrm{Au}^{\prime}$ species. As shown here, the excess of 4-PSH can be significantly reduced and the reaction rate markedly accelerated by adding external $\mathrm{S}_{8}$, which under optimized conditions leads to very fast and quantitative dissolution of $A u\left(0.10 \mathrm{~mol} /\left(\mathrm{m}^{2 *} \mathrm{~h}\right)\right)$.

The reaction mechanism is supported by identification of intermediate species and rationalized by quantum-chemical calculations. The success with 4-PSH ligand is a balanced combination of its good coordinative ability with respect to $\mathrm{Au}^{0}$ and the low oxidation potential of the generated bisligand intermediate. As a result of one-electron oxidation, a soluble $\mathrm{Au}^{\prime}$ complex is formed with sulfate counter anion. Notably, the studies with 2-PSH and 2-MBIH ligands illustrate how the dissolution of 
$\mathrm{Au}^{0}$ is markedly hindered either by higher formation energies of the reactive bisligand intermediates or by their higher oxidation potentials.

The 4-PSH dissolution method was also applied to $\mathrm{Cu}^{0}$ and $\mathrm{Ag}^{0}$ substrates resulting in high dissolution efficiencies. The method possesses inherent selectivity towards metals which undergo one-electron oxidation; $\mathrm{Pt}$ and $\mathrm{Pd}$ thin films remain intact during the 4-PSH treatment. Furthermore, selectivity within Group 11 was demonstrated by changing the ligand; 2-MBI as a reactive ligand prefers only $\mathrm{Cu}^{0}$. This selectivity results from the remarkably lower formation energy of the bisligand intermediate $\mathrm{Cu}(2-\mathrm{MBI})_{2}$ in comparison to the $\mathrm{Au}$ and $\mathrm{Ag}$ species. The possibility to dissolve coinage metals with benign and mild methods is highly important for human endeavors towards sustainable society and circular economy. In this respect, the dissolution was further implemented as an efficient method for Au films and printed circuit board samples. Thus, the principles learned from the studies reported herein could find future applications in sustainable dissolution pathways for noble metals.

\section{Methods}

Standard Au dissolution reaction. Au powder (2 mg, $10 \mu \mathrm{mol})$ and 4-PSH (226 mg, $2 \mathrm{mmol}$ ) were weighted in a glass vial $(20 \mathrm{ml})$ followed by addition of DMF $(5 \mathrm{ml})$. The reaction mixture was stirred at $\mathrm{RT}$ for a few minutes to dissolve all 4-PSH. Then $\mathrm{H}_{2} \mathrm{O}_{2}$ (33\% in water, $92.8 \mu \mathrm{L}, 1 \mathrm{mmol}$ ) was added ( $\mathrm{Au} / 4-\mathrm{PSH} / \mathrm{H}_{2} \mathrm{O}_{2}$ molar ratio 1:200:100) and the reaction was placed in an oil bath set to $60{ }^{\circ} \mathrm{C}$ and stirred for $8 \mathrm{~min}$. Samples for FAAS analysis were prepared by diluting the reaction mixture in DMF $(300 \mu \mathrm{l} / 9.5 \mathrm{ml})$.

\section{References}

1. Freakley, S., He, Q. \& Kiely, C. Gold Catalysis: A Reflection on Where We are Now. Catal. Lett. 145, 71-79 (2015).

2. Corti, C. W. \& Holliday, R. J. Commercial Aspects of Gold Applications: From Materials Science to Chemical Science. Gold. Bull. 37, 20-26 (2004).

3. Steele, B. C. H. \& Heinzel, A. Materials for Fuel-Cell technologies. Nature 414, 345-352 (2001).

4. Zhang, J., Sasaki, K., Sutter, E. \& Adzic, R. R. Stabilization of Platinum Oxygen-Reduction Electrocatalysts using Gold Clusters. Science 315, 220-222 (2007).

5. Hagelüken, C. \& Corti, C. W. Recycling of gold from electronics: Cost-effective use through 'Design for Recycling'. Gold Bull. 43, 209-220 (2010).

6. Hilson, G. \& Monhemius, A. J. Alternatives to cyanide in the mining industry: what prospects for the future? J. Cleaner Prod. 14, 1158-1167 (2006).

7. Behnamfard, A., Salarirad, M. M \& Veglio, F. Process development of recovery of copper and precious metals from waste printed circuit boards with emphasis on palladium and gold leaching and precipitation. Waste Manage. 33, 2354-2363 (2013). 
8. Lin, W., Zhang, R.-W., Jang, S.-S., Wong, C.-P. \& Hong, J.-I. "Organic Aqua Regia"-Powerful Liquids for Dissolving Noble Metals. Angew. Chem. Int. Ed. 49, 7929-7932 (2010).

9. Lin, W. Recovery of high-purity Pt from Pt-Au bimetallic nanoparticles using organic aqua regia. Rare Metals 31, 92-95 (2012).

10. Lin, W. Organic Aqua Regia: Discovery, Fundamentals, and Potential Applications. Noble Metals, 335-352 (2012).

11. Serpe, A., Artizzu, F., Mercuri, M. L., Pilia, I. \& Deplano, P. Charge transfer complexes of dithioxamides with dihalogens as powerful reagents in the dissolution of noble metals. Coord. Chem. Rev. 252, 1200-1212 (2008).

12. Bigoli et al. A powerful new oxidation agent towards metallic gold powder: N,N'dimethylperhydrodiazepine-2,3-dithione (D) bis(diiodine). Synthesis and X-ray structure of $\left[A u D I_{2}\right] l_{3}$. Chem. Commun. 2351-2352 (1998).

13. Isaia et al. Gold oxidative dissolution by (thioamide)- $I_{2}$ adducts. Dalton Trans. 42, 492-498 (2013).

14. Cau et al. New powerful reagents based on dihalogen/N,N'-dimethylperhyrdodiazepine-2,3dithione adducts for gold dissolution: the IBr case. Dalton Trans., 1969-1974 (2003).

15. Serpe, A., Marchiò, L., Artizzu, F., Mercuri, L. \& Deplano, P. Effective One-Step Gold Dissolution Using Environmentally Friendly Low-Cost Reagents. Chem. Eur. J. 19, 10111-10114 (2013).

16. Räisänen, M. T., Kemell, M., Leskelä, M. \& Repo, T. Oxidation of elemental gold in alcohol solutions. Inorg. Chem. 46, 3251-3256 (2007).

17. Cometto, F. P., Macagno, V. A., Parades-Olivera, P. \& Patrito, E. M. Decomposition of methylthiolate monolayers on $\mathrm{Au}(111)$ prepared from dimethyl disulfide in solution phase. J. Phys. Chem. C, 114, 10183-10194 (2010).

18. Schoenfisch, M. H. \& Pemberton, J. E. Air stability of alkanethiol self-assembled monolayers on silver and gold surfaces. J. Am. Chem. Soc., 120, 4502-4513 (1998).

19. Jia et al. New thiocyanatocadmates templated by multi-dentate $\mathrm{N}$-heterocyclic/diamine molecules. Dalton Trans. 42, 6429-6439 (2013).

20. $\mathrm{Yu}$ et al. Structural characterization of layered double salt $\mathrm{Mn}_{3}(\mathrm{OH})_{2}\left(\mathrm{SO}_{4}\right)_{2}\left(\mathrm{H}_{2} \mathrm{O}\right)_{2} \cdot \mathrm{K}_{2} \mathrm{SO}_{4}$. Inorg. Chem. Commun. 10, 159-162 (2007).

21. Salah et al. Synthesis, nuclear, and magnetic structures and magnetic properties of $\left[\mathrm{Mn}_{3}(\mathrm{OH})_{2}\left(\mathrm{SO}_{4}\right)_{2}\left(\mathrm{H}_{2} \mathrm{O}\right)_{2}\right]$. Chem. Eur. J. 10, 2048-2057 (2004).

22. Salah, M. B., Vilminot, S., Mhiri, T. \& Kurmoo, M. Synthesis, crystal structure, and magnetic properties of $\mathrm{Mn}_{2}(\mathrm{OH})_{2} \mathrm{SO}_{4}$ : a novel layered hydroxide. Eur. J. Inorg. Chem. 2272-2276 (2004).

23. Wade, L. Gold's Dark Side. Science 341, 1448-1449 (2013).

24. Serpe, A., Artizzu, F., Espa, D., Rigoldi, A., Mercuri, M. \& Deplano, P. From trash to resource: a green approach to noble-metals dissolution and recovery. Green Process Synth. 3, 141-146 (2014). 


\section{Acknowledgements}

The financial support of the Academy of Finland is gratefully acknowledged. M.M. thanks Professor Filipp Furche for hosting and his support in the computational studies by the National Science Foundation under $\mathrm{CHE}-1464828$.

\section{Author contributions}

M.T.R. and E.H. contributed equally and produced most of the experimental results and analyzed the data. M.M. performed computational studies. M.K. prepared Ag and Pd substrates and carried out the FESEM-EDS measurements and J.H. prepared Pt substrates. M.T.R. and M.N. carried out the crystallographic characterization and K.M. performed ESI-MS experiments. F.A.-Q., A.E. and H.L. contributed to dissolution experiments and K.L. assisted in preparation of the manuscript. T.R. supervised the project. All the authors have agreed to all the content of the manuscript.

\section{Data availability}

Supplementary information and chemical compound information are available in the online version of the paper. Correspondence and requests for material should be addressed to T.R. and those related to computational studies to M.M. CCDC 1852214 (1) contains the supplementary crystallographic data for this paper. These data can be obtained free of charge from The Cambridge Crystallographic Data Centre via www.ccdc.cam.ac.uk/data request/cif.

\section{Competing financial interests}

The authors declare no competing financial interests. 